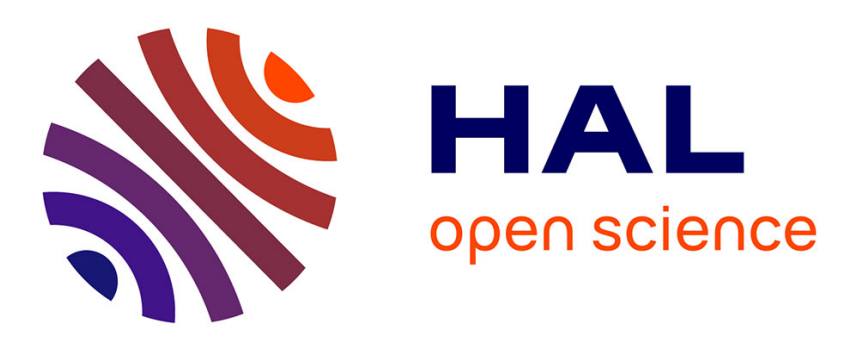

\title{
Haptic Rendering of Interacting Dynamic Deformable Objects Simulated in Real-Time at Different Frequencies
}

François Dervaux, Igor Peterlik, Jérémie Dequidt, Stéphane Cotin, Christian Duriez

\section{- To cite this version:}

François Dervaux, Igor Peterlik, Jérémie Dequidt, Stéphane Cotin, Christian Duriez. Haptic Rendering of Interacting Dynamic Deformable Objects Simulated in Real-Time at Different Frequencies. IROS 2013 - IEEE/RSJ International Conference on Intelligent Robots and Systems, Nov 2013, Tokyo, Japan. hal-00842866

\section{HAL Id: hal-00842866 https://hal.inria.fr/hal-00842866}

Submitted on 9 Jul 2013

HAL is a multi-disciplinary open access archive for the deposit and dissemination of scientific research documents, whether they are published or not. The documents may come from teaching and research institutions in France or abroad, or from public or private research centers.
L'archive ouverte pluridisciplinaire HAL, est destinée au dépôt et à la diffusion de documents scientifiques de niveau recherche, publiés ou non, émanant des établissements d'enseignement et de recherche français ou étrangers, des laboratoires publics ou privés. 


\title{
Haptic Rendering of Interacting Dynamic Deformable Objects Simulated in Real-Time at Different Frequencies
}

\author{
Francois Dervaux ${ }^{1}$, Igor Peterlik ${ }^{2}$, Jérémie Dequidt ${ }^{1}$, Stéphane Cotin ${ }^{1}$, Christian Duriez $^{1}$
}

\begin{abstract}
The dynamic response of deformable bodies varies significantly in dependence on mechanical properties of the objects: while the dynamics of a stiff and light object (e.g. wire or needle) involves high-frequency phenomena such as vibrations, much lower frequencies are sufficient for capturing dynamic response of an object composed of a soft tissue. Yet, when simulating mechanical interactions between soft and stiff deformable models, a single time-step is usually employed to compute the time integration of dynamics of both objects. However, this can be a serious issue when haptic rendering of complex scenes composed of various bodies is considered.

In this paper, we present a novel method allowing for dynamic simulation of a scene composed of colliding objects modelled at different frequencies: typically, the dynamics of soft objects are calculated at frequency about $50 \mathrm{~Hz}$, while the dynamics of stiff object is modeled at $1 \mathrm{kHz}$, being directly connected to the computation of haptic force feedback. The collision response is performed at both low and high frequencies employing data structures which describe the actual constraints and are shared between the high and low frequency loops. During the simulation, the realistic behaviour of the objects according to the mechanical principles (such as non-interpenetration and action-reaction principle) is guaranteed. Examples showing the scenes involving different bodies in interaction are given, demonstrating the benefits of the proposed method.
\end{abstract}

\section{INTRODUCTION}

Real-time simulation of deformable bodies is an intensive area of research in the field of computer graphics, virtual reality and haptics. Among applications targeted by the research, there is a strong need for surgical training systems based on high-fidelity haptic interaction with simulated deformable objects. An important advances have taken place recently, often being related to utilization of the finite element method (FEM) that allows for accurate modeling of mechanical behaviour observed in physical objects. If interaction equiped with force feedback is to be considered, the fidelity of the feedback is significantly influenced by the accuracy of deformation modeling, but also by the performance of algorithms employed for modeling the interactions (such as contacts, grasping).

Mainly in the area of haptic rendering, the main challenge remains the computation of these deformations and interactions in real-time. Although the finite element method is an elegant tool for modeling the dynamic behaviour of objects, it remains computationally expensive for complex objects with irregular geometries that appear in medical simulations.

\footnotetext{
*This work was supported by French Research Agency with the program IDeaS (ANR JCJC SIMI 3).

${ }^{1}$ Shacra Team-Project, INRIA Lille - Nord-Europe and University Lille 1 - LIFL CNRS UMR 8022, France francois. dervauxeinria.fr

${ }^{2}$ IHU Strasbourg, 67000 Strasbourg, France
}

Several strategies have been adopted to accelerate the computations, either by simplifying the simulation of the object behavior, or the interaction taking place among the modeled objects.

The dynamic response may vary significantly depending on geometric and material characteristics of the object: for example let us suppose a simulation scenario where the scene is composed of two objects: a flexible needle interacting with an deformable organ such as liver. Clearly, as the needle is stiff and light, realistic simulation of its dynamic response requires high frequencies to be taken into account, whereas in the case of the soft tissue which is bulky and soft much lower frequencies are needed to capture its response correctly. However, the temporal integration used in this scenario usually relies on single time step in simulation of both objects, mainly if an interaction between the two bodies is modelled in the simulation. Such an approach can be acceptable in the case where the simulation is not required to be run in a real-time: in this case a small simulation time step is chosen to account for the high frequencies in the needle response. Thereby, it's assumed that it will run much more slowly than the real clock time, as in each small time step, the costly dynamics of the bulky soft objects has to be recomputed.

Such a solution is not sustainable any more, as soon as the simulation is to be run in real-time. In this context, we focus on a multi-rate approach where dynamics of each object in the scene is integrated in real-time with its own time step. We believe that this approach is especially suited for simulations with haptic rendering. In this case, the dynamics of the stiff object (e.g. the flexible needle) which does not require heavy computations can be simulated in the haptic loop running usually at $1000 \mathrm{~Hz}$, while the dynamics of the soft highly-deformable body (e.g. the liver) is simulated on a much lower (typically synchonized with the visual thread running about 30 or $60 \mathrm{~Hz}$ ), since it requires more computationally expensive calculations. This approach is already studied for example in [1] where the objects are simulated at different frequencies, however, at the cost of simplification in modeling the interactions.

The work presented in this paper is based on [2] where a methods allowing for multi-rate interactive simulation of objects simulated at two different frequencies has been proposed. However, the method was suitable only for a quasi-static scenario: as it required that compliance matrices needed to simulate the interactions between the objects do not depend on time step. The contribution of this paper is to overcome this limitation and extend the multirate approach to 
constraint-based interaction to dynamic scenario. To our best knowledge, this is the first approach that allows for real-time dynamic simulation of colliding deformable objects, whose dynamics is computed at different frequencies.

The remainder of this paper is organized as follows: a brief overview of related work is given in section 2 . Then the multi-rate dynamics is presented in section 3 mainly from the mathematical point of view. In section 4 , the computational model is presented and finally the results are presented and discussed in section 5.

\section{Previous Works}

Haptic rendering is a productive and very active research field and the literature on the topic is quite vast. We narrow the scope of this section to research focusing on stable realistic haptic rendering of contacts between soft deformable bodies and the works dealing with asynchronous and/or multi-rate methods.

Haptic interaction with deformable bodies is based on two competing but coupled scientific problems: realistic soft body simulation which is computationally intensive and requires large integration time steps in order to be interactive, and realistic haptic rendering which implies fast computations of forces and therefore requires small integration time steps. In order to achieve an efficient trade-off, works in this field usually rely on two main simplification strategies to achieve the realistic haptic rendering: either the mechanical coupling between the simulation scene and the haptic device is simplified or the complexity of the mechanical models is reduced substantially.

Pioneering works in the field employed the first approach by allowing for local interactions only. For instance BroNielsen and Cotin [3] use bilateral constraints solved with Lagrange Multipliers in order to prevent inter-penetrations whereas Popescu and Compton [4] introduce small area paradigm which defines equality constraints that will impact the position of a reduced set of surface vertices of the deformable surface. Unilateral contact combined with computation of capacitance matrix is introduced by James and Pai[5] using pre-computed Green's functions that is valid for linear deformations. These methods are limited to an interaction with single contact point.

Handling complex and multiple friction contacts is introduced by Duriez et al. [6] but the method also requires the pre-computation of a compliance matrix and the method is also limited to linear deformations. Extension to non-linear deformations is proposed by Barbic and James [7] that allows for multiple contacts and self-collisions of soft bodies with penalty forces. As penalty can not reproduce precise contact and friction conditions, Saupin et.al [8] propose to use an approximations of the compliance that allows to handle multiple friction contact points using constraints on nonlinear models. Similarly, Garre and Otaduy [9] only consider the block-diagonal matrix of the compliance matrix assuming small displacements between time steps. These later works enable more advanced haptic interaction but are based on assumptions that are not compatible with realistic modeling of dynamic response in stiff and light deformable objects.

Recent works propose computationally fast deformable models in order to achieve high-frequency simulation. For instance, approach proposed by Barbic and James [7] is compatible with reduced deformation models. Similar approach is used in the work of Jacobs et. al. [10] where a fast linearized deformable model is computed.

Multi-rate or asynchronous simulation has also been much investigated the past few years in order to handle computationally expensive deformable models. Harmon et. al. [11] describe a multi-rate simulation framework that prevents inter-penetrations, obeys causality and guarantees the conservation of momentum and energy. Their work relies, among other contributions, on asynchronous variational integrators and kinematic data structures. While proven to complete in finite time, such framework is not intended for haptic interactions where small and strictly bounded computation time step is required. More recent works have addressed the performance issue of multi-rate simulation. Efficient culling of collisions [12] or intermediate representations called phantom meshes [13] have been investigated to reduce the computation burden. Nevertheless, the proposed methods are still far from providing a stable haptic rendering of complex objects. Others works deal with haptic interactions provided by dynamic simulation, but are still limited to rigid [14] objects or textiles [15].

The main objective of our approach is to provide realistic haptic rendering of deformable models that can handle a large scope of soft bodies (from stiff and light objects to soft and heavy ones). Our approach combines a multirate simulation and an accurate mechanical coupling. The major improvement over the previous work in [2] lies in the capability of our framework to handle full second-order dynamics and is no more restricted to quasi-static scenario.

\section{DYNAMIC AND ASYNCHRONOUS DEFORMABLE MODELS IN CONTACT}

This section presents an overview of our asynchronous framework and details the mathematical notation that will be used throughout the paper. More particularly, we detail the models and the numerical methods involved in the simulation of dynamic deformable solids simulated at different frequencies. Additionally, we present the constraint-based solving process associated to the mechanical interactions between these deformable objects.

Our framework relies on simulation loops running at different frequencies. To simplify the explanations and without limiting the generality of the approach, we consider that we have two simulation loops for simulating the interactions between two deformable solids. The first loop, named low rate loop, is dedicated to the computation of the dynamics of the object that can be simulated with a large integration time-step (e.g. $20 \mathrm{~ms}$ ). The solid with such a low dynamics is relatively heavy and composed of soft material. We call it the soft object. 
The second one, named high rate loop, handles the simulation and the haptic rendering with a small integration time-step, typically $1 \mathrm{~ms}$ in order to compute correctly the dynamics of the deformations of a light and stiff object. This solid is called the stiff object.

The following notation is used throughout the remaining of the paper: for any mechanical variable, such as $\mathbf{M}$, the right subscript indicates the corresponding object, the left subscript represents which simulation loops computed the value ( $L$ for the low rate loop, $H$ for the high rate one) and the left superscript which integration time-step has been used to compute the mechanical variable. In our framework, two integration steps are used $\Delta T$ which is the larger time-step and $d t$ which is the smaller time-step. For instance ${ }_{L}^{d t} \mathbf{M}_{\text {stiff }}$ encodes the mass matrix of the object named stiff. This matrix is computed in the low rate simulation loop using an integration time-step of $d t$. The same notation is consistently derived to represent vectors: ${ }_{L}^{d t} \mathbf{v}_{\text {soft }}$ is the velocity vector of the object soft being computed in the low rate loop using an integration time-step of $d t$.

\section{A. FEM-based deformable models}

The deformable models used in our simulation are based on the Finite Element Method (FEM). The materials are supposed to have linear elastic constitutive laws but geometric non-linearities due to large transformations are handled. As FEM models are generally computationally expensive, it is worth mentioning the requirement that the computation of the deformations must be included in the two simulation loops.

Thus, the model used for the stiff solid must have a very quick computation method to hold the real-time constraint. In our work we have used serially linked beam elements that can fulfill the computation time requirements [16] while accounting for non-linear deformations. On the contrary, the computation time for the soft object is less constrained. Typically, we can use a volume FEM model composed of several hundreds of tetrahedral elements. To capture the geometrical nonlinearities, we rely on a corotational formulation [17].

In both cases, the FEM models are composed of elements that integrate the material properties of the solids (mass, elasticity) and of nodes which are the degrees of freedom sampled over the deformable domains. Both deformable solids behave according to the equation of the dynamics:

$$
\mathbf{M a}=\mathbf{f}(\mathbf{x}, \mathbf{v})+\mathbf{J}^{T} \lambda
$$

where a denotes the acceleration of the nodes, $\mathbf{x}$ and $\mathbf{v}$ are respectively the node positions and velocities, $\mathbf{M}$ is the mass matrix and $\mathbf{f}$ is the sum of internal stiffness forces and external forces. Finally, the second term of the right-hand member of Eq.(1) stands for the constraints imposed on the solid: $\mathbf{J}^{T}$ provides the directions of constraints and $\lambda$ is the vector of constraint response forces.

The time integration is performed using a backward Euler integration scheme. Implicit integration is preferred to ensure stable behavior regardless the choice of the time-steps. Denoting $h$ the time-step used in the integration ( $h=d t$ for small time step and $h=\Delta T$ for large time step), the backward Euler scheme provides the following equations:

$$
\mathbf{v}^{t+h}=\mathbf{v}^{t}+h \mathbf{a}^{t+h} \quad \mathbf{x}^{t+h}=\mathbf{x}^{t}+h \mathbf{v}^{t+h} .
$$

The forces $\mathbf{f}$ are non-linear functions of the positions $\mathbf{x}$ and velocities $\mathbf{v}$. We apply a Taylor series expansion to $\mathbf{f}$ and make the first order approximation (a single linearization by time step):

$$
\mathbf{f}\left(\mathbf{x}^{t+h}, \mathbf{v}^{t+h}\right) \approx \mathbf{f}\left(\mathbf{x}^{t}, \mathbf{v}^{t}\right)+\mathbf{K}\left(\mathbf{x}^{t+h}-\mathbf{x}^{t}\right)+\mathbf{B}\left(\mathbf{v}^{t+h}-\mathbf{v}^{t}\right)
$$

where $\mathbf{f}$ represents the internal stiffness forces at a given position $\mathbf{x}$ of the degrees of freedom, $\mathbf{K}$ is the stiffness matrix depending on the current position. A damping matrix $\mathbf{B}=\alpha \mathbf{M}+\beta \mathbf{K}$ is introduced in the Eq.3, where $\alpha$ and $\beta$ are respectively the Rayleigh mass and the Rayleigh damping coefficients. By combining equations (1) and (3) we get

$$
\underbrace{\left(\frac{1}{h} \mathbf{M}-\mathbf{B}-h \mathbf{K}\right)}_{\mathbf{A}} \mathbf{d} \mathbf{v}=\underbrace{\mathbf{f}\left(\mathbf{x}^{t}, \mathbf{v}^{t}\right)+h \mathbf{K} \mathbf{v}^{t}}_{\mathbf{b}}+\frac{1}{h} \mathbf{J}^{t} \lambda
$$

where $\mathbf{d v}$ is defined as $h \mathbf{a}=\mathbf{v}^{t+h}-\mathbf{v}^{t}$. The matrix $\mathbf{A}$ is refreshed at each time step. We emphasize that the matrix A depends on the time step $h$, so the matrix is not the same with $h=d t$ and with $h=\Delta T$.

\section{B. Interaction Model}

The FEM models of both stiff and soft objects are linked by the constraint based expression of their mechanical interactions. Among the interactions between the two deformable bodies, we need to detect the collision and provide an adequate response (without interpenetration). The collision detection (or more generally the geometrical determination of the contact points) is often time consuming and is not compatible with the high rate loop. In our approach, we use an algorithm based on proximity queries between FEM meshes that is computed in the low rate loop. It supposes that we have access to a geometrical position of the stiff object in this low rate loop. The algorithm places contact constraints where the local minimal distance between the meshes is small enough to have a potential collision between current time $t$ and $t+\Delta T$. Other type of constraints, for instance bilateral constraints (attachment, sliding..) can also be set. After computing the queries, a matrix $\mathbf{J}$ containing the directions of unilateral and bilateral constraints for each object is assembled.

To handle the contacts realistically, we rely the Signorini's law $^{1} 0 \leq \boldsymbol{\delta} \perp \boldsymbol{\lambda} \geq 0$ where $\boldsymbol{\delta}$ represents the signed distances between potential contact points detected by the proximity queries. The bilateral constraints prevent the relative motion of the bodies at a given point, in a given direction. Thus, at this point the relative distance is forced to $\delta=0$. The constraints are set in the low rate loop but a very important feature of our method is that the resolution of the constraints is performed at both high and low rates, in order to have adequate constraint response on both dynamic models.

\footnotetext{
${ }^{1}$ We can also set additional constraints to simulate the friction with Coulomb's law like it is done in [6].
} 
To solve the reaction forces of this response, we first to need evaluate the constraint violation when applying an unconstrained dynamic motion to both deformable objects (i.e. solving Eq.(4) with $\boldsymbol{\lambda}=0$.). These unconstrained motions are called the free motions. Computed on the stiff and soft objects with respectively small $h=d t$ and large $h=\Delta T$ time steps, they provide the positions ${ }_{H}^{d t} \mathbf{x}_{\text {stiff }}^{\text {free }}$ and ${ }_{L}^{\Delta T} \mathbf{X}_{\text {soft }}^{\text {free }}$. However, to obtain a measure of the violation for both $h=d t$ and $h=\Delta T$, we also need to compute a value for ${ }_{L}^{d t} \mathbf{x}_{\text {soft }}^{\text {free }}$ and ${ }_{L}^{\Delta T} \mathbf{x}_{\text {stiff }}^{\text {free }}$ (i.e. the free position for the soft object with $h=d t$ and for the stiff object with $h=\Delta T$ ).

Obtaining these position values require to solve two additional linear system of equation. This is not a problem for the stiff solid because the cost of solving its system at low rates can be neglected. In the contrary, for the soft object, its system can be very large. Thus, the value ${ }_{L}^{d t} \mathbf{x}_{\mathrm{soft}}^{\mathrm{free}}$ is computed at low rates. This approximation is valid because the soft body has a low dynamics. If it were computed at high rate, the displacement created by the unconstrained motion ${ }_{H}^{d t} \mathbf{x}_{\text {soft }}^{\text {free }}-{ }_{H}^{d t} \mathbf{x}_{\text {soft }}$ would not change much between high rate steps .

When obtaining the violation $\delta^{\text {free }}$ at both low and high rates, we need to solve, at low and high rates a mixed complementarity problem (MCP). First we compute the MCP at low rates, with a time step $\Delta T$ :

$$
\left\{\begin{array}{l}
\boldsymbol{\delta}=\underbrace{\left[{ }_{L}^{\Delta T} \mathbf{W}_{\text {soft }}+{ }_{L}^{\Delta T} \mathbf{W}_{\text {stiff }}\right]}_{{ }^{\Delta T} \mathbf{W}} \lambda{ }^{\Delta T} \boldsymbol{\delta}^{\text {free }} \\
0 \leq \boldsymbol{\delta} \perp \boldsymbol{\lambda} \geq 0 \quad \text { (for contact constraints) } \\
\boldsymbol{\delta}=0 \quad \text { (for bilateral constraints) }
\end{array}\right.
$$

where the compliance matrices for soft and stiff objects are computed as

$$
\begin{array}{rll}
{ }_{L}^{\Delta T} \mathbf{W}_{\text {soft }} & ={ }_{L} \mathbf{J}_{\text {soft }}{ }_{L}^{\Delta T} \mathbf{A}_{\text {soft }}^{-1} & { }_{L} \mathbf{J}_{\text {soft }}^{T} \\
{ }_{L} T \mathbf{W}_{\text {stiff }} & ={ }_{L} \mathbf{J}_{\text {stiff }}{ }_{L}^{\Delta T} \mathbf{A}_{\text {stiff }}^{-1} & { }_{L} \mathbf{J}_{\text {stiff }}^{T}
\end{array}
$$

Again, as it was already the case for the free motion, we need to compute the compliance matrix of the stiff object with large time steps $h=\Delta T$ in order to have an adequate response of the constraints at low rates. This is important as for dynamic systems, the value of the matrix $\mathbf{A}$ defined at Eq.(4) depends on the time step $h$.

For the MCP at high rates, we use the violation of the constraints ${ }_{H}^{d t} \delta^{\text {free }}$ based on the positions obtained at high rates. Additionally, we compute the compliance matrices obtained with small time step $h=d t$. For the stiff object, the matrix ${ }_{H}^{d t} \mathbf{A}_{\text {stiff }}$ is refresh at high rates, and as the object has a fast dynamic response, important changes in ${ }_{H}^{d t} \mathbf{A}_{\text {stiff }}$ can be observed between two small time step $d t$. However, we do the hypothesis that the directions of constraints in $\mathbf{J}_{\text {stiff }}$ can be provided by the low rate loop (in order to avoid an update of the proximity queries algorithm). This is valid only if these directions do not change much in the high rate loop between two updates. Thus, the computation of the compliance matrix for the stiff object is:

$$
{ }_{H}^{d t} \mathbf{W}_{\text {stiff }}={ }_{L} \mathbf{J}_{\text {stiff }}{ }_{H}^{d t} \mathbf{A}_{\text {stiff }}^{-1}{ }_{L} \mathbf{J}_{\text {stiff }}^{T}
$$

It must be emphasized that the computational cost needed for this matrix must be compatible with the real-time constraint on the high rate loop.

Finally, we need to compute the compliance ${ }^{d t} \mathbf{W}_{\text {soft }}$ that would have the soft object if it were integrated with small time steps $h=d t$. The computation of this matrix is expensive as it involves the inverse of the matrix $\mathbf{A}_{\text {soft }}$ which is not optimized for being updated at high rates. But as the soft object has a low dynamic response, we estimate that ${ }^{d t} \mathbf{A}_{\text {soft }}$ does not change between two time steps computed at low rates. Thus, the computation of the compliance matrix ${ }^{d t} \mathbf{W}_{\text {soft }}$ is performed at low rates:

$$
{ }_{L}^{d t} \mathbf{W}_{\text {soft }}={ }_{L} \mathbf{J}_{\text {soft }}{ }_{L}^{d t} \mathbf{A}_{\text {soft }}^{-1} \mathbf{J}_{\text {soft }}^{T} .
$$

Finally, if the values computed at low rates are shared with the high rates loop, we can compute the MCP at high rates:

$$
\left\{\begin{array}{l}
\boldsymbol{\delta}=\underbrace{\left[{ }_{L}^{d t} \mathbf{W}_{\text {soft }}+{ }_{H}^{d t} \mathbf{W}_{\text {stiff }}\right]}_{{ }_{L}^{d t} \mathbf{W}} \boldsymbol{\lambda}+{ }_{H}^{d t} \boldsymbol{\delta}^{\text {free }} \\
\begin{array}{l}
0 \boldsymbol{\delta} \perp \boldsymbol{\lambda} \geq 0 \quad \text { (for contact constraints) } \\
\boldsymbol{\delta}=0 \quad \text { (for bilateral constraints) }
\end{array}
\end{array}\right.
$$

The resolution of this MCP provides an adequate response for the constraints on the stiff object simulated at high rates.

Overall, to build the $2 \mathrm{MCPs}$, three compliance matrices are computed at low rates (two matrices with two different time-steps $d t$ and $\Delta T$ for the soft object and one matrix with time step $\Delta T$ for the stiff object). One compliance matrix is computed at high rates for the stiff object with the small time step $d t$. Consequently it can be observed that the method provides additional computations at low rates for obtaining adequate compliance matrices, but these computations can be easily parallelized.

When the MCP are solved, a corrective motion can be applied to each object. It consist in solving Eq.(4) with the new value of $\boldsymbol{\lambda}$ and with $\mathbf{b}=0$. Then, as the system in Eq.(4) is linear, we can compute the final motion by adding the free motion and the corrective motion.

\section{Computational Model}

In this part, the computational model showing the implementation of two threads and structures shared between them is given in detail. The symbols introduced in the previous section are employed to denote the vectors and matrices corresponding to the objects simulated at different frequencies.

\section{A. Multi-rate algorithm for dynamic modeling}

The simulation is performed in two separated threads each running at different frequency. The first thread implements the high rate loop of the simulation in which the dynamics of the stiff object is computed and directly coupled to the haptic force feedback. In the second thread, the dynamics of the soft object is modeled, together with full resolution of the LCP being in charge of constraint resolution as described in section III-B. 


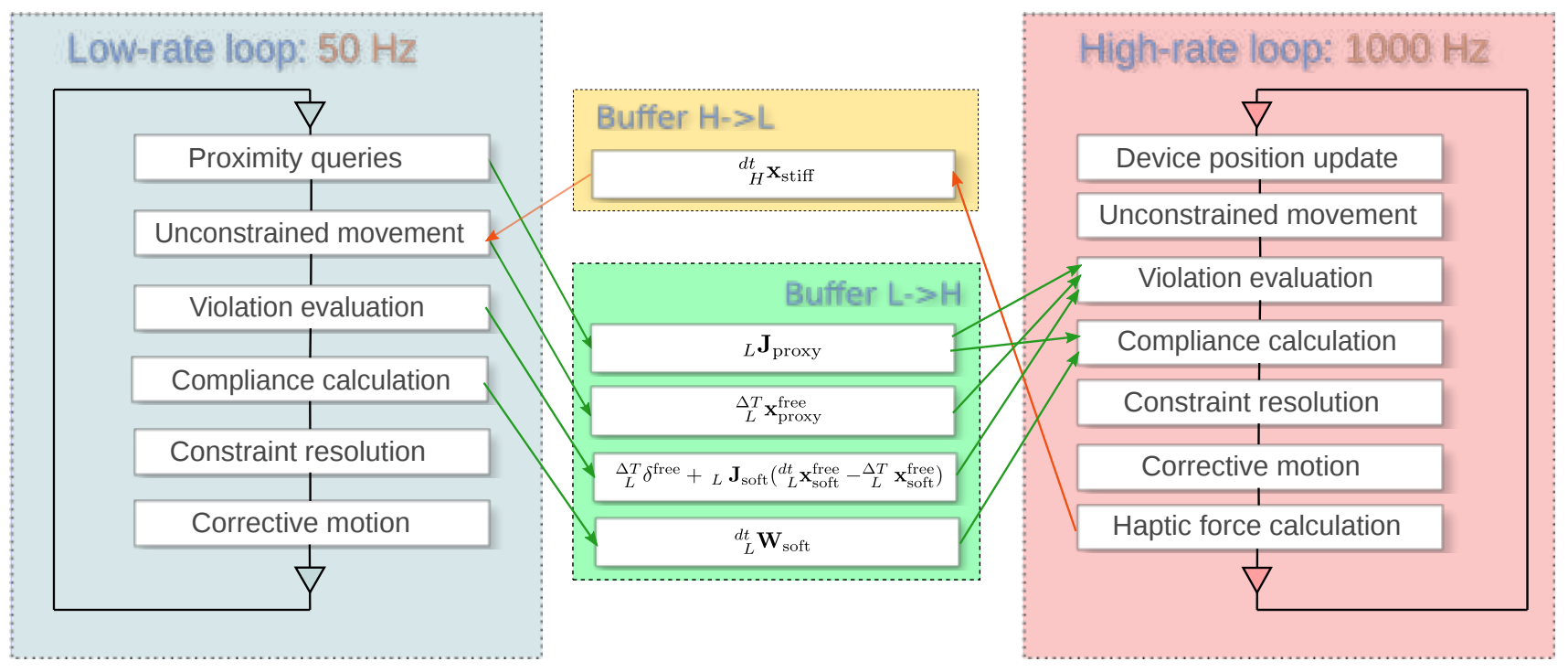

Fig. 1. Schematic visualization of the computational model.

In each time-step of the low rate loop, seven sub-steps L1 - L7 are performed as follows:

L1 Potential collisions are detected using proximity queries which are evaluated using the position vectors ${ }_{L} \mathbf{x}_{\text {proxy }}$ and ${ }_{L} \mathbf{x}_{\text {soft }}$ computed in the last sub-step of the previous time-step. The calculation results in matrices ${ }_{L} \mathbf{J}_{\text {proxy }}$ and ${ }_{L} \mathbf{J}_{\text {soft }}$. Both matrices are stored to the buffer to be available to the high rate loop.

L2 The position of the stiff object ${ }_{H} \mathbf{x}_{\text {stiff }}$ computed in the sub-step $\mathrm{H6}$ of the previous high rate loop time-step is imposed to the proxy.

L3 The unconstrained dynamics of each object is simulated: first, using $\Delta T$ for both proxy and soft, the system matrices (4) are assembled and inverted resulting in ${ }_{L}^{\Delta T} \mathbf{A}_{\text {proxy }}^{-1}$ and ${ }_{L}^{\Delta T} \mathbf{A}_{\text {soft }}^{-1}$ and unconstrained positions are computed and stored in vectors ${ }_{L} T \mathbf{x}_{\text {proxy }}^{\text {free }},{ }_{L} T \mathbf{X}_{\text {soft }}^{\text {free }}$. Second, the calculation is repeated for the soft object, however, high rate time-step $d t$ is used instead of $\Delta T$ to compute the system matrix, resulting in inverted matrix ${ }_{L}^{d t} \mathbf{A}_{\text {soft }}^{-1}$ and unconstrained position vector ${ }_{L}^{d t} \mathbf{x}_{\text {soft }}^{\text {free }}$.

L4 The constraint violation ${ }_{L}^{\Delta T} \boldsymbol{\delta}^{\text {free }}$ is computed as shown in section III-B. An additional correction term is computed as ${ }_{L} \mathbf{J}_{\text {soft }}\left({ }_{L}^{d t} \mathbf{x}_{\text {soft }}^{\text {free }}-{ }_{L}{ }_{L} T \mathbf{X}_{\text {soft }}^{\text {free }}\right)$. This term is necessary for the calculation of constraint violation in the high rate loop as shown in $\mathrm{H} 3$. Both the violation and corrective term vectors are stored in the buffer.

L5 Two compliance matrices, ${ }_{L}^{\Delta T} \mathbf{W}_{\text {proxy }}$ and ${ }_{L}^{\Delta T} \mathbf{W}_{\text {soft }}$ are computed for each object using ${ }_{L}^{\Delta T} \mathbf{A}_{\text {proxy }}^{-1}$ and ${ }_{L}^{\Delta T} \mathbf{A}_{\text {soft }}^{-1}$. One compliance matrix ${ }_{L}^{d t} \mathbf{W}_{\text {soft }}$ is computed for the soft object using ${ }^{d t} \mathbf{A}_{\text {soft }}^{-1}$. The latter is stored to the buffer.

L6 The constraint resolution is performed in the low rate loop using the compliance ${ }_{L}^{\Delta T} \mathbf{W}={ }_{L}^{\Delta T} \mathbf{W}_{\text {soft }}+{ }_{L}^{\Delta T} \mathbf{W}_{\text {stiff }}$ and the vector ${ }_{L}^{\Delta T} \boldsymbol{\delta}^{\text {free }}$ computed in L4., resulting in the vector of corrective forces $\frac{\Delta T}{L} \boldsymbol{\lambda}$.

L7 Finally, the force vector ${ }_{L}^{\Delta T} \boldsymbol{\lambda}$ is used to apply the motion correction to both proxy and the soft objects resulting in constrained positions ${ }_{L}^{\Delta T} \mathbf{x}_{\text {proxy }}$ and ${ }_{L}^{\Delta T} \mathbf{x}_{\text {soft }}$.

The high rate simulation loop running in parallel performs 6 sub-steps in one time-step employing the data buffered in the last time-step of the low rate loop as follows:

H1 The position representing the location of the haptic interaction point is updated according to the actual position of the haptic device.

$\mathrm{H} 2$ The unconstrained position ${ }_{H}^{d t} \mathbf{x}_{\text {stiff }}^{\text {free }}$ of the stiff object is computed using the inverse ${ }_{H}^{d t} \mathbf{A}_{\text {stiff }}^{-1}$ of the system matrix.

H3 The constraint violation ${ }_{H}^{d t} \boldsymbol{\delta}^{\text {free }}$ is computed. It is not possible to use directly the violation ${ }_{L}^{\Delta T} \delta^{\text {free }}$ calculated in L4 of the low rate loop, since this vector was computed from unconstrained position simulated with a different time-step $\Delta T$. Nevertheless, the desired high rate violation can be obtained as follows:

$$
\begin{aligned}
{ }_{H}^{d t} \boldsymbol{\delta}^{\text {free }} & ={ }_{L}^{\Delta T} \boldsymbol{\delta}^{\text {free }} \\
& +{ }_{L} \mathbf{J}_{\text {soft }}\left({ }_{L}^{d t} \mathbf{x}_{\text {soft }}^{\text {free }}-{ }_{L} T \mathbf{x}_{\text {soft }}^{\text {free }}\right) \\
& +{ }_{L} \mathbf{J}_{\text {proxy }}\left({ }^{d t}{ }_{H} \mathbf{x}_{\text {stiff }}^{\text {free }}-{ }_{L} T \text {. } \mathbf{x}_{\text {proxy }}^{\text {free }}\right)
\end{aligned}
$$

where (11) is a corrective term compensating for the different time-step in soft object dynamics computed in L4 and (12) is a similar corrective term computed here using the unconstrained positions of the proxy and stiff objects calculated in L3 and $\mathrm{H} 2$, respectively.

H4 The compliance matrix ${ }_{H}^{d t} \mathbf{W}$ is computed as a sum of ${ }_{L}^{d t} \mathbf{W}_{\text {soft }}$ computed in L5 and

$$
{ }_{H}^{d t} \mathbf{W}_{\text {stiff }}=\left\{{ }_{L} \mathbf{J}_{\text {proxy }}\right\}\left\{{ }_{H}^{d t} \mathbf{A}_{\text {stiff }}^{-1}\right\}\left\{{ }_{L} \mathbf{J}_{\text {proxy }}^{\top}\right\} .
$$

H5 The time-limited constraint resolution is performed using ${ }_{H}^{d t} \mathbf{W}$ from $\mathrm{H} 4$ and ${ }_{H}^{d t} \boldsymbol{\delta}^{\text {free }}$ from H3, resulting in correction force vector ${ }_{H}^{d t} \boldsymbol{\lambda}$. 


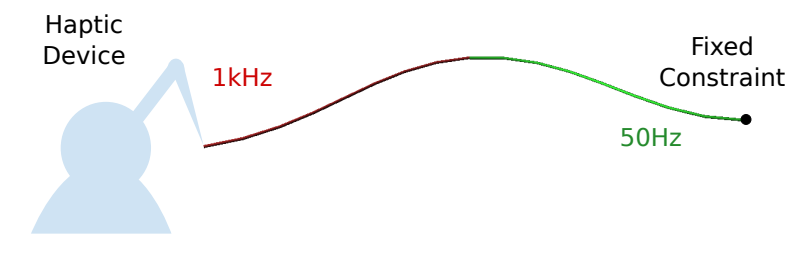

Fig. 2. Illustration of the two beams example. Deformations are the same at low rates (green) and at high rates (red).

H6 The forces stored in ${ }_{H}^{d t} \boldsymbol{\lambda}$ are used to compute the corrected position ${ }_{H}^{d t} \mathbf{x}_{\text {stiff }}$ of the stiff object. The position ${ }_{H}^{d t} \mathbf{x}_{\text {stiff }}$ is stored to the buffer to be available for the low rate proxy object needed in sub-step L2.

\section{B. Force-feedback for haptic rendering}

As described in the previous, the dynamics of the stiff body is computed in the high rate loop as realistic simulation of its behaviour requires small time-step. Moreover, this body usually represents the object (such as the deformable needle) which is driven by the user. However, this means that this tool is attached to the haptic device and the force response of the tool represents the force-feedback which should be delivered to the user via the haptic device.

In our setting, the vector ${ }_{H}^{d t} \lambda$ computed in $\mathrm{H} 5$ gathers the actual forces corresponding to the constraints imposed on both soft and stiff objects. In order to compute the response force in the nodes of the stiff object corresponding to the constraint forces, the constraint forces has to be transformed from the constraint space to the space of degrees of freedom of the stiff object. This is done using the matrix ${ }_{L} \mathbf{J}_{\text {proxy }}$ which contains the constraint directions computed in substep L1 as $\mathbf{f}_{\text {stiff }}=\left\{{ }_{L} \mathbf{J}_{\text {proxy }}\right\}_{H}^{d t} \boldsymbol{\lambda}$ where $\mathbf{f}_{\text {stiff }}$ is the vector of forces applied in the nodes of the stiff object due to the constraints imposed on the stiff object. Finally, the force feedback is calculated by summing the vector $\mathbf{f}_{\text {stiff }}$ over the nodes of the stiff object, i.e. $\mathbf{f}_{\text {haptic }}=\sum_{n} \mathbf{f}_{\text {stiff }}^{n}$.

\section{Results}

In this section we present results of experiments demonstrating the multi-rate method presented in this paper.

The first experiments highlight the action-reaction principle. We propose a basic case which allows to verify that the principle is preserved in simulation implemented by our method. Two beam models with identical mechanical parameters are connected with a bilateral constraint. The other end of the low rate beam model is fixed in the space, whereas the free end of the high rate beam model is attached to the haptic device. The experiment show that although being computed on different frequencies, the beams undergoes symmetric deformations for an arbitrary position of the haptic device after the simulation is stabilized. Moreover, the dynamics of the high rate beam allows for high rate vibrations observed during the simulation.
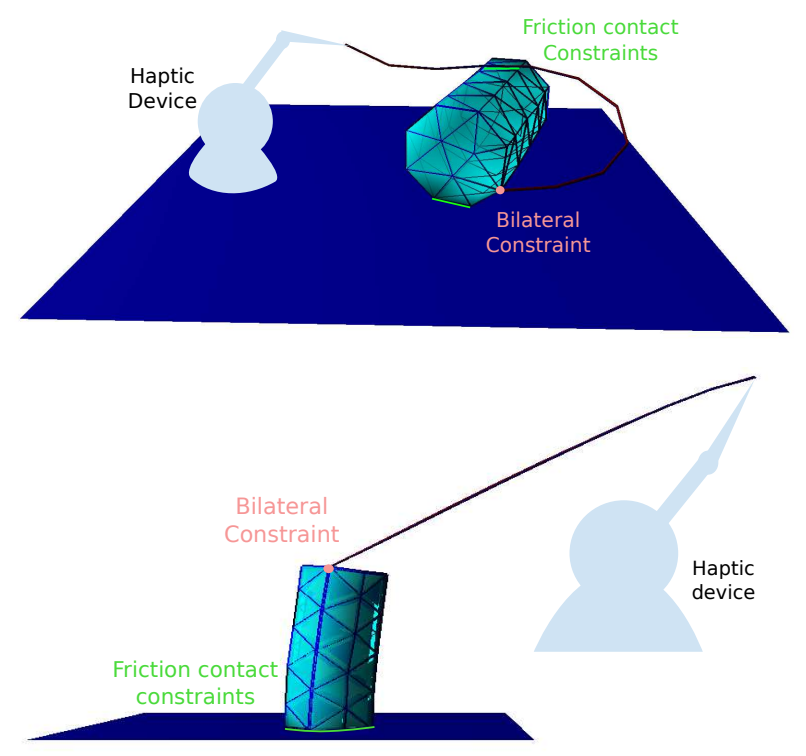

Fig. 3. Illustration of the scene composed by a cylinder (simulated at $50 \mathrm{~Hz}$ ) and a thread (simulated at $1 \mathrm{kHz}$ ).

To validate our approach, we created another simulation scene where a cylinder is placed on a horizontal plane. The cylinder is modelled by tetrahedral corotational FEM and is simulated at $50 \mathrm{~Hz}$. A wire is attached to the upper part of the cyliner and the other endpoint of the thread is driven by a haptic device as shown in Fig. 3. The wire is modelled with 10 serially-linked beam elements based on Tymoshenko formulation and its dynamics is computed at $1000 \mathrm{~Hz}$. The collisions between the plane, cylinder and the thread are being detected and resolved during the simulation. During the interaction, the simulation remains stable for any admissible configuration: the position of the cylinder on the plane can be changed by pulling the wire, or the cylinder can be even pulled out of the plane, so it's hanging freely in the space been hung on the wire. At the same time, a detailed and realistic haptic force-feedback is delivered to the user via the mechanism described in section IV-B.

\section{CONCLUSIONS}

In this paper a significant issue in haptic rendering of deformable object is addressed. The main contribution of the paper is in proposing a method that allows for dynamic simulation of deformable objects being computed at different frequencies: while the collisions between the objects are handled correctly, a stable haptic feedback is delivered to the user. While additional computational overhead is introduced in the low rate loop in which three compliance matrices are computed, the calculations in the high rate thread remain compatible with frequency of $1000 \mathrm{~Hz}$ required by the haptic rendering.

Examples are provided demonstrating that the actionreaction principle is well preserved in the simulation and further, the high rate response allows for correct rendering of such phenomena as high-frequency vibration of the thread, 
which rapidly increases quality of haptic rendering of light and stiff objects such as wires and needles.

In the paper, a scenario employing two different frequencies is presented. While we believe such scenario already opens a wide possibilities for many applications in virtual reality, extending our approach to scenario with more than two frequencies should be investigated. Simulations that include deformable objects with motion computed at their optimal frequency would enable more complex simulations without sacrificing the realism, accuracy and quality of dynamic response. However, scaling our approach to many simulation frequencies may drastically increase the computational burden as well as the volume of data shared between the simulation loops. Therefore future work will aim at providing a generic way to handle multi-rate simulations while reducing the computational overhead to handle the interactions.

\section{REFERENCES}

[1] J. Dequidt, L. Grisoni, and C. Chaillou, "Collaborative interactive physical simulation," in GRAPHITE '05: Proceedings of the 3rd international conference on Computer graphics and interactive techniques in Australasia and South East Asia, S. Spencer, Ed. ALCOVE (INRIA / LIFL), Dec. 2005, pp. 147-150. [Online]. Available: http://is.gd/DqSgXg

[2] I. Peterlik, C. Duriez, and S. Cotin, "Asynchronous haptic simulation of contacting deformable objects with variable stiffness," in Intelligent Robots and Systems (IROS), 2011 IEEE/RSJ International Conference on, Sept., pp. 2608-2613.

[3] M. Bro-Nielsen and S. Cotin, "Real-time volumetric deformable models for surgery simulation using finite elements and condensation," Computer Graphics Forum, vol. 15, no. 3, pp. 57-66, 1996.

[4] D. C. Popescu and M. Compton, "A model for efficient and accurate interaction with elastic objects in haptic virtual environments," in GRAPHITE '03: Proceedings of the 1st international conference on Computer graphics and interactive techniques in Australasia and South East Asia, 2003, pp. 245-250.

[5] D. L. James and D. K. Pai, "A unified treatment of elastostatic contact simulation for real time haptics," in SIGGRAPH '05: ACM SIGGRAPH 2005 Courses, 2005, p. 141.

[6] C. Duriez, F. Dubois, A. Kheddar, and C. Andriot, "Realistic haptic rendering of interacting deformable objects in virtual environments," IEEE Transactions on Visualization and Computer Graphics, vol. 12, no. 1, pp. 36-47, 2006.

[7] J. Barbič and D. L. James, "Six-dof haptic rendering of contact between geometrically complex reduced deformable models," IEEE Trans. Haptics, vol. 1, no. 1, pp. 39-52, 2008.

[8] G. Saupin, C. Duriez, and S. Cotin, "Contact model for haptic medical simulations," in ISBMS '08: Proceedings of the 4th international symposium on Biomedical Simulation. Berlin, Heidelberg: SpringerVerlag, 2008, pp. 157-165.

[9] C. Garre and M. A. Otaduy, "Haptic rendering of complex deformations through handle-space force linearization," World Haptics Conference, vol. 0, pp. 422-427, 2009.

[10] P. Jacobs, M. J. Fu, and M. C. Cavusoglu, "High Fidelity Haptic Rendering of Frictional Contact with Deformable Objects in Virtual Environments using Multi-rate Simulation," The International Journal of Robotics Research, Sept. 2010. [Online]. Available: http://ijr.sagepub.com/cgi/doi/10.1177/0278364910378540

[11] D. Harmon, E. Vouga, B. Smith, R. Tamstorf, and E. Grinspun, "Asynchronous contact mechanics," ACM Transactions on Graphics, vol. 28, no. 3, p. 1, July 2009. [Online]. Available: http://portal.acm.org/citation.cfm?doid=1531326.1531393

[12] S. Ainsley, E. Vouga, E. Grinspun, and R. Tamstorf, "Speculative parallel asynchronous contact mechanics," ACM Trans. Graph., vol. 31, no. 6, pp. 151:1-151:8, Nov. 2012. [Online]. Available: http://doi.acm.org/10.1145/2366145.2366170
[13] D. Harmon, Q. Zhou, and D. Zorin, "Asynchronous integration with phantom meshes," in Proceedings of the 2011 ACM SIGGRAPH/Eurographics Symposium on Computer Animation, ser. SCA '11. New York, NY, USA: ACM, 2011, pp. 247-256. [Online]. Available: http://doi.acm.org/10.1145/2019406.2019439

[14] I. Susa, M. Sato, and S. Hasegawa, "Multi-rate multi-range dynamic simulation for haptic interaction," in World Haptics Conference (WHC), 2011 IEEE, June, pp. 233-238.

[15] G. Böttcher, D. Allerkamp, and F.-E. Wolter, "Multirate coupling of physical simulations for haptic interaction with deformable objects," The Visual Computer, vol. 26, no. 6-8, pp. 903-914, Apr. 2010. [Online]. Available: http://www.springerlink.com/index/10.1007/s00371-010-0450-1

[16] C. Duriez, S. Cotin, J. Lenoir, and P. F. Neumann, "New approaches to catheter navigation for interventional radiology simulation," Computer Aided Surgery, vol. 11, no. 6, pp. 300-308, 2006.

[17] C. Felippa and B. Haugen, "A unified formulation of small-strain corotational finite elements: I. theory," Computer Methods in Applied Mechanics and Engineering, vol. 194, no. 21, pp. 2285-2335, 2005. 